\title{
CD26 Expression on T-Anaplastic Large Cell Lymphoma (ALCL) Line Karpas 299 is associated with increased expression of Versican and MT1-MMP and enhanced adhesion
}

\author{
Pamela A Havre', Long H Dang ${ }^{1}$, Kei Ohnuma², Satoshi Iwata², Chikao Morimoto² and Nam H Dang ${ }^{1,3^{*}}$
}

\begin{abstract}
Background: CD26/dipeptidyl peptidase IV (DPPIV) is a multifunctional membrane protein with a key role in T-cell biology and also serves as a marker of aggressive cancers, including T-cell malignancies.

Methods: Versican expression was measured by real-time RT-PCR and Western blots. Gene silencing of versican in parental Karpas 299 cells was performed using transduction-ready viral particles. The effect of versican depletion on surface expression of MT1-MMP was monitored by flow cytometry and surface biotinylation. CD44 secretion/ cleavage and ERK (1/2) activation was followed by Western blotting. Collagenase I activity was measured by a live cell assay and in vesicles using a liquid-phase assay. Adhesion to collagen I was quantified by an MTS assay.

Results: Versican expression was down-regulated in CD26-depleted Karpas 299 cells compared to the parental T-ALCL Karpas 299 cells. Knock down of versican in the parental Karpas 299 cells led to decreased MT1-MMP surface expression as well as decreased CD44 expression and secretion of the cleaved form of CD44. Parental Karpas 299 cells also exhibited higher collagenase I activity and greater adhesion to collagenase I than CD26-knockdown or versican-knockdown cells. ERK activation was also highest in parental Karpas 299 cells compared to CD26-knockdown or versican-knockdown clones.

Conclusions: Our data indicate that CD26 has a key role in cell adhesion and invasion, and potentially in tumorigenesis of T-cell lines, through its association with molecules and signal transduction pathways integral to these processes.
\end{abstract}

Keywords: CD26, T-cell malignancies, Adhesion, MT1-MMP, Cell signaling

\section{Background}

CD26/dipeptidyl peptidase IV (DPPIV) is a $110-115 \mathrm{kD}$ glycosylated protein that exists as a homodimer. It is a multifunctional membrane protein with three domains: extracellular, transmembrane, and cytoplasmic. It is widely expressed on a number of tissues and can regulate tumor growth and development [1-7]. The interaction of CD26/ DPPIV with other proteins, including collagen, fibronectin, and caveolin-1, likely influences its involvement in cell

\footnotetext{
* Correspondence: nam.dang@medicine.ufl.edu

'Division of Hematology/Oncology, University of Florida Shands Cancer Center, Gainesville, FL 32610, USA

${ }^{3}$ Division of Hematology/Oncology, University of Florida, 1600 SW Archer Road, Box 100278, Gainesville, Florida 32610, USA

Full list of author information is available at the end of the article
}

motility and invasion [8,9]. CD26 and its associated DPPIV enzyme activity play a key role in T-cell biology, serving as a marker of T-cell activation and participating in several signaling pathways [10-13]. CD26 is also a marker of aggressive cancers, including T-cell malignancies [14-20]. Interestingly, the cleaved form of CD26, which is present in plasma, is inversely correlated with several aggressive cancers [21].

Our previous work showed that CD26-depleted human T-anaplastic large cell lymphoma (T-ALCL) Karpas 299 cells were unable to form tumors in SCID mice [8], and that CD26 expression on two T-cell lines increased SDF-1- $\alpha$-mediated invasion [22]. We were interested in looking at CD26-associated gene products involved in 
cell motility and therefore conducted microarray analysis of genes involved in this pathway in parental Karpas 299 and CD26-depleted clones, and found that versican expression was associated with changes in CD26 level. Microarray analysis revealed that mRNA level for versican was considerably lower in CD26-depleted Karpas 299 cells than parental Karpas 299 cells (1:88). Although mRNA levels for several other genes, including IGFBP3, tenascin C, and SPOCK1, were also lower in CD26depleted cells than parental Karpas 299, Western blots confirmed a difference in protein expression for versican only, but not for the other three proteins. Versican is a large chondroitin sulfate proteoglycan involved in the regulation of adhesion, migration, invasion, and angiogenesis [23]. Versican binds to ECM constituents including type I collagen, fibronectin, and hyaluronan (HA) [24] and a number of cell-surface proteins, including CD44, integrin $\beta 1$, and toll receptor $2[25,26]$. Versican levels are elevated in most malignancies, and correlated with poor patient outcome. Versican is secreted by peritumoral stromal cells and also by the individual cancer cells $[27,28]$. Four major isoforms exist that differ with respect to the number and position of GAG molecules attached, which are important for association with other proteins. Of note is that the V0 and V1 isoforms are reported to be the isoforms most closely associated with cancers.

In the present paper, we examined in detail CD26 involvement with cell migration and adhesion in T-cell lines. Expression array analyses of genes involved in extracellular matrix and adhesion pathways indicated that versican expression was significantly higher in parental T-ALCL Karpas 299 cells compared to CD26depleted Karpas 299 cells. To further investigate the relationship between CD26 and versican, we conducted knock down studies of versican in Karpas 299 cells and evaluated for a potential effect on expression of signaling proteins and adhesion. We found that the use of shRNA to knock down versican expression in the parental Karpas 299 cells resulted in both lower MT1-MMP transcription and surface expression. To confirm that cell behavior was consistent with the observed change in MT1-MMP activity, several assays were performed; secretion and cleavage of CD44, collagenase I activity, and adhesion. In all three assays, parental Karpas 299 cells exhibited higher activity compared to cells in which CD26 or versican was knocked down. Finally, ERK activation, which is required for migration and invasion, was also highest in the parental Karpas 299 cell line.

\section{Methods \\ Reagents}

Bovine serum albumin (BSA), polybrene (hexadimethrine bromide), sodium dodecyl sulfate, glycine, sodium deoxycholate, trypsin, phosphate buffered saline, and dimethyl sulfoxide were from Sigma Life Science, St. Louis, MO. TX-100, NP-40, and Tween-20 were from Fisher Scientific, USA. Puromycin was from Life Technologies, USA. Rat tail collagen and bovine skin collagen were purchased from BD and Advanced Matrix, respectively. GM6001, a general MMP inhibitor was purchased from Calbiochem.

\section{Cell culture}

Karpas 299 cells were originally obtained from the American Type Culture Collection (ATCC, Manassas, VA) and maintained in RPMI-1640 (Hyclone, Logan, UT). Karpas 299 cells depleted of CD26 have been described previously [8]. All cell media contained 10\% fetal bovine serum (Hyclone), penicillin $(100 \mathrm{u} / \mathrm{ml})$ and streptomycin $(100 \mu \mathrm{g} / \mathrm{ml})$.

\section{Expression arrays}

GEArray express human extracellular matrix and adhesion molecule microarrays were carried out by SuperArray Bioscience Corporation on $10 \mu \mathrm{g}$ total RNA isolated from parental Karpas 299 cells and Dep1, a cell line deficient in CD26 expression.

\section{Real-time RT-PCR}

Real-time RT-PCR was carried out on $10 \mathrm{ng}$ total RNA (RNeasy kit, Qiagen). SYBR Green-based real-time RT-PCR was carried out using QuantiTect Primer Assays (Qiagen) for CD26 (Hs_DPP4_1_SG), Versican (Hs_VCAN_1_SG), and GAPDH (Hs_GAPDH_1_SG).

\section{RT-PCR}

RT-PCR was carried out on 10 ng of RNA isolated from parental Karpas 299 cells, Dep1, and Dep2 using the Titan One Tube RT-PCR system (Roche Applied Science). The primers were described previously [29]. The sizes of the amplification products were $405 \mathrm{bp}$ for $\mathrm{V} 0$ (forward: 5' - TCAACATCTCATGTTCCTCCC-3' and reverse: 5'-TTC TTCACTGTGGGTATAGGTCTA-3') and $336 \mathrm{bp}$ for V1 (forward: 5' -GGCTTTGACCAGTGC GATTAC-3' and reverse: 5' -TTCTTCACTGTGGGTA TAGGTCTA-3'). The reverse transcription step was carried out at $50^{\circ}$ for $30 \mathrm{~min}$, followed by denaturation for $2 \mathrm{~min}$ at $94^{\circ}$, amplified by 35 cycles $\left(94^{\circ}\right.$ for $30 \mathrm{~s}, 55^{\circ}$ for $45 \mathrm{~s}, 68^{\circ}$ for $45 \mathrm{~s}$ ) and elongated for $7 \mathrm{~min}$ at $68^{\circ}$.

\section{Flow cytometry}

Cells were washed once with staining buffer (PBS containing 1\% BSA) and incubated on ice for 30 minutes with antibodies specific for the activity domain of MT1MMP (ab51074, Abcam, Cambridge, MA), then with FITC goat anti-rabbit Ig at $0.125 \mu \mathrm{g} / 10^{6}$ cells (BD Pharmingen). After washing with staining buffer twice, the 
cells were resuspended in PBS. The optimum amount of MT1-MMP antibody was determined by titration.

\section{Gene silencing}

Transduction ready viral particles for gene silencing of versican (versican shRNA, Santa Cruz Biotechnology, Inc., Santa Cruz, CA) were used to infect Karpas cells at a ratio of 0.5 virus particles per cell. Cells were pelleted the following day, resuspended in fresh media, and $48 \mathrm{hrs}$ following transduction, puromycin was added at a concentration of $2.5 \mathrm{ug} / \mathrm{ml}$. Following selection, stable clones were isolated by limiting dilution. Knockdown was monitored by running whole cell lysates and/or spent media on gels and probing with versican antibodies as described in the Western Blot section.

\section{Cell lysis}

Cells were lysed using RIPA (1\% NP40, 0.5\% DOC, 0.1\% SDS, $150 \mathrm{mM} \mathrm{NaCl}, 50 \mathrm{mM}$ TrisCl, pH 8.0) or TX100 buffer (50 mM TrisCl, pH 8, $0.15 \mathrm{M} \mathrm{NaCl}, 1 \%$ TX-100) containing a protease/phosphatase inhibitor cocktail (Pierce, Rockford, IL). Protein concentration was determined using the bicinchoninic acid protein assay reagent (Pierce).

\section{Isolation of vesicles from serum free media}

Cells $\left(8 \times 10^{6}\right)$ were grown in serum free media for 48 hours, followed by centrifugation at $600 \times \mathrm{g}$ for $15 \mathrm{~min}$, then $1500 \times \mathrm{g}$ for $15 \mathrm{~min}$, and the resulting supernatant was subsequently centrifuged at 100,000 $\times \mathrm{g}$ for $1 \mathrm{hr}$ at $4^{\circ} \mathrm{C}$. Pelleted vesicles were suspended in PBS and assayed for protein [30].

\section{Western blots}

Equal amounts of protein were run on $5.0,7.5 \%$ or $10 \%$ polyacrylamide gels. For detection of versican, samples were combined with sample buffer without reducing agent. Following transfer, blots were blocked, then probed with one of the following antibodies: anti-CD26 (AF1180) and anti-CD44H (clone 2C5) were from R \& $\mathrm{D}$ Systems, Inc., Minneapolis, $\mathrm{MN}$; anti-versican (clone 2B1, Seikagaku, Tokyo, Japan); and anti-MT1-MMP (ab38971, Abcam). Anti-phospho-p44/42 MAPK (Erk 1/2) and anti-p44/42 MAPK (Erk $1 / 2$ ) were from Cell Signaling Technology, Inc; anti-integrin alpha 5 chain (BD, cat\# 610633). Precision Plus Protein Standards (Bio-Rad Laboratories, Hercules, CA) were run to estimate sizes of proteins of interest. Horseradish peroxidase-conjugated secondary antibodies and the detection reagent, SuperSignal West Dura Extended Duration Substrate, were from Pierce. Films were scanned using an Image Quant 400 (GE Healthcare, Piscataway, NJ).

\section{Biotinylation and immunoprecipitation}

Cells were suspended in PBS $(2.5 \times 107 / \mathrm{ml})$ and incubated with $200 \mu \mathrm{l}$ of $10 \mathrm{mM}$ EZ-Link ${ }^{\otimes}$ Sulfo-NHS-LCBiotin/ml cells for $30 \mathrm{~min}$ on ice. The cells were then washed $3 \times$ with PBS containing $100 \mathrm{mM}$ glycine. Following lysis in TX100 buffer, $1 \mathrm{mg}$ lysate was applied to a Streptavidin- Agarose spin column (Pierce), and following extensive washing, bound proteins were eluted with $2 \times$ sample buffer and heating at $100^{\circ} \mathrm{C}$ for $5 \mathrm{~min}$. Eluates were run on $7.5 \%$ acrylamide gels and probed with anti-MT1-MMP antibody.

\section{Collagen degradation in cultured cells}

Collagen I degradation was monitored in live cells migrating through a native $3 \mathrm{D}$ collagen substrate. $\mathrm{DQ}^{\mathrm{TM}}$ collagen, type I from bovine skin, fluorescein conjugate (Molecular Probes) was copolymerized with rat-tail collagen type I, in RPMI media without phenol red (Life Technologies). After incubation for $48 \mathrm{hrs}$ at $37^{\circ} \mathrm{C}$, solid phase collagen and cells were pelleted and the supernatant analyzed for FITC using a Perkin-Elmer Victor ${ }^{3}$ $\mathrm{V}$ multilabel counter [31].

\section{Collagen degradation in vesicles}

The EnzChek collagenase assay (Life Technologies) was used to evaluate activity in vesicles isolated from conditioned media. In this assay, $\mathrm{DQ}^{\mathrm{mi}}$ collagen, type I from bovine skin, fluorescein conjugate (Molecular Probes) was used as substrate and the incubation was carried out at room temperature as described by the manufacturer. Each well of a 96 well plate contained $4.5 \mu \mathrm{g}$ vesicle protein. Fluorescence was detected using the Perkin-Elmer instrument.

\section{Adhesion assays}

Adhesion assays were carried out essentially as described [8]. Cells $\left(5 \times 10^{5} /\right.$ well $)$ were seeded into 12 well collagen I coated plates and incubated overnight. Unattached cells were removed, plates were washed three times with PBS and the adhesive cells remaining were quantified using the MTS assay. The total cell number was determined using uncoated wells and serial dilutions were used to construct a standard curve to convert absorbance at $490 \mathrm{~nm}$ to cell number.

\section{Results}

Model showing idealized scheme for interaction of signaling molecules in parental Karpas 299 cells

Figure 1 depicts a simplified scheme for molecules believed to be involved in CD26 enhanced invasion. In this proposed model for parental Karpas 299 cells, CD26 is shown bound to the cell membrane. Results from our microarray analysis indicated that in CD26-depleted cells, versican was underexpressed, at a ratio of 1:80 compared 


\section{Karpas 299 parental cell}

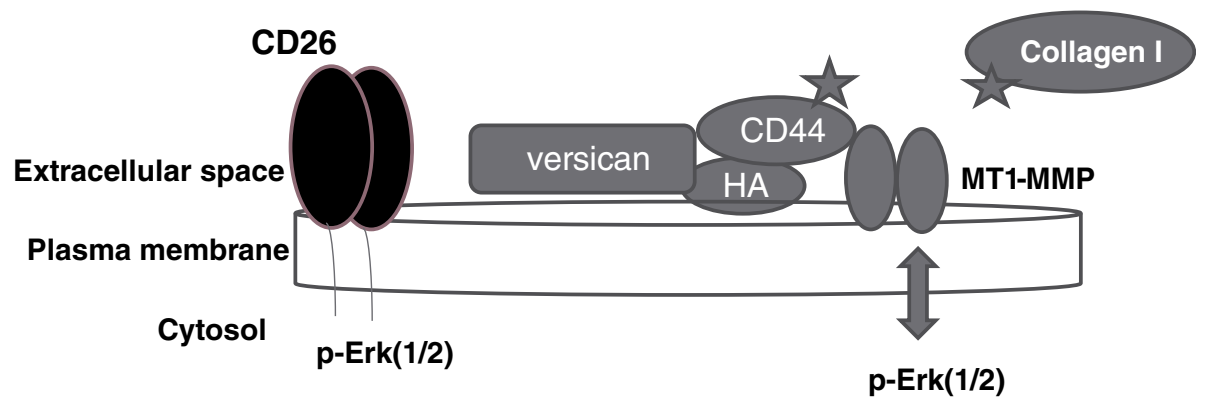

Figure 1 Model for CD26 regulation of adhesion and downstream signaling. In this simplified scheme, CD26 is shown bound to the cell membrane. Versican is also depicted in the membrane, but is also secreted and is a constituent of the extracellular matrix. CD44 and HA are bound to versican, but CD44 is also bound to MT1-MMP, which can itself cleave CD44, resulting in CD44 secretion. Secretion of the cleaved CD44 is necessary for localization of MT1-MMP at the invadopodia where it digests collagen I, a constituent of the extracellular matrix. In addition, Erk (1/2) activation occurs in the parental Karpas cells and has been reported to be required for migration, invasion, and CD44 upregulation. This model is intended to be a working hypothesis of the relationship between the proteins shown here.

to the parental cell. Versican is an extracellular matrix component and is involved in diverse activities, including adhesion, proliferation, migration, and angiogenesis. MT1MMP is a membrane MMP and is also involved in these activities. It is one of the few MMPs that can degrade directly collagen I, a component of the extracellular matrix. CD44 binds to both versican and MT1-MMP, which is able to cleave CD44. It is thought that cleavage and release of CD44 from the membrane is required for the relocalization of MT1-MMP to the invadopodia, where it binds to collagen I, leading to invasion of the extracellular matrix. Relocation to the invadosome may occur in vesicles (or exosomes). Activation of Erk (1/2) is also shown here, since it is reported to form a positive feedback loop with MT1-MMP and has been shown to regulate invasive activity.

Decreased expression of versican is associated with CD26 depletion in human T-anaplastic large cell lymphoma Karpas 299

Our previous work showed that depletion of CD26 in Karpas 299 cells resulted in loss of cell adhesion to the extracellular matrix and decreased tumorigenicity in a SCID mouse xenograft model [8]. To identify CD26associated gene products potentially involved in cell adhesion processes, we performed expression microarray analysis of human extracellular matrix and adhesion molecules with RNA isolated from parental Karpas 299 and the CD26-depleted Karpas 299 cell line Dep1 [8]. Our data indicated that expression of versican was approximately 90-fold higher in the parental Karpas 299 cells compared to CD26-depleted Karpas 299 cells (Table 1).
Real-time RT-PCR and Western blots were subsequently carried out to confirm differential expression of versican in parental Karpas 299 cells and the two CD26depleted Karpas 299 cell lines Dep1 and Dep2 [8]. RNA was isolated from Karpas 299, Dep1, and Dep2 cells, and SYBR Green based real-time RT-PCR was performed using QuantiTect Primer Assays. Down-regulation of versican was confirmed in CD26 depleted cells, with an 80-fold and 103-fold enrichment for parental Karpas 299 compared to Dep1 and Dep2, respectively (Table 2). Western blot analyses also confirmed that versican expression was higher in parental Karpas 299 as compared to Dep1 and Dep2 (Figure 2A). RT-PCR using V0 and V1 specific primers were used to confirm this as shown in Figure 2B.

\section{Enhanced expression of MT1-MMP is associated with CD26 and versican in Karpas 299}

MT1-MMP (MMP14) plays a critical role in the process of cell motility and invasion, with its deletion in tumor cells resulting in the loss of both in vitro and in vivo invasive activity [32]. We therefore examined its status in parental Karpas 299 and the CD26-depleted Karpas 299

Table 1 Oligo GE Array microarrays indicate that versican mRNA expression is higher in CD26-expressing cells than in CD26-depleted cells (Dep1)

\begin{tabular}{lccccc}
\hline Unigene & RefSeqNo & Symbol & Dep1 & Karpas & Karpas/Dep1 \\
\hline Hs.544577 & NM_002046 & GAPDH & 253.7 & 141.5 & 0.56 \\
Hs.443681 & NM_004385 & VCAN & 0.68 & 60.12 & 88.4
\end{tabular}

GEArray express human extracellular matrix and adhesion molecule microarrays were carried out by SuperArray Bioscience Corporation on $10 \mu \mathrm{g}$ total RNA isolated from parental Karpas 299 cells and Dep1, a cell line deficient in CD26 expression. 
Table 2 Real-time RT-PCR was used to confirm Versican expression

\begin{tabular}{lccc}
\hline GAPDH & Avg Ct & Karpas/Dep1 & Karpas/Dep2 \\
\hline Karpas & 17.74 & - & - \\
Dep1 & 16.70 & 0.49 & - \\
Dep2 & 16.72 & - & 0.49 \\
CD26 & & & - \\
Karpas & 20.93 & - & - \\
Dep1 & 23.95 & 8.11 & 8.69 \\
Dep2 & 24.05 & - & - \\
Versican & & - & - \\
Karpas & 25.51 & 80 & 103 \\
Dep1 & 31.83 & - & \\
Dep2 & 32.20 & - &
\end{tabular}

RNA was isolated from Karpas 299 cells and two clones, Dep1 and Dep2, in which CD26 is depleted. SYBR Green-based real-time RT-PCR was carried out on 10 ng total RNA using QuantiTect Primer Assays for CD26, Versican, and GAPDH.

Dep1 and Dep2 cell lines. In addition, to further evaluate the effect of versican depletion in the T-ALCL Karpas 299 cell line independent of CD26 status, we established a number of versican knock down Karpas 299 lines, as described in Materials and Methods and shown in Figure 2.

Since only MT1-MMP expressed on the cell surface mediates degradation of the extracellular matrix [32], we next evaluated its surface expression by both cell surface biotinylation and flow cytometry analysis, as described in Materials and Methods. Cells were cultured overnight in collagen I coated wells to stimulate MT1-MMP expression [33]. Our data indicated that a higher percentage of parental Karpas 299 cells exhibited surface expression of MT1-MMP than CD26-depleted Dep1 or versican-knock down clone 6RD3 (Figure 3A).

Meanwhile, flow cytometry studies also demonstrated that the presence of collagen induced greater surface expression of MT1-MMP in all cells tested (Figure 3B). Importantly, a higher percentage of parental Karpas 299 cells expressed surface MT1-MMP than Dep1 or 6RD3 clones in the presence or absence of collagen. Of note is the fact that our experiments consistently found MT1-MMP to be expressed at relatively low levels on the cell surface, findings which were consistent with previous work demonstrating that only small amount of MT1-MMP is expressed on the cell surface at any one time [34].

\section{Enhanced CD44 expression is associated with CD26 and versican in Karpas 299}

MT1-MMP has been reported to associate with several membrane-associated and cytosolic proteins, including CD44 [35]. Interaction of MT1-MMP with CD44 leads to the cleavage of CD44 and facilitates migration by indirectly linking MT1-MMP to the cytoskeleton [35,36]. Our present work demonstrated that expression of CD44 in total cell lysates (Figure 4A) and secretion of its cleaved form in conditioned media (Figure 4B) were higher in parental Karpas 299 as compared to the CD26depleted Dep1 and versican-depleted 1A12 and 6RD3 clones. Since PMA has been shown to increase CD44 expression [37] and to stimulate trafficking of MT1-

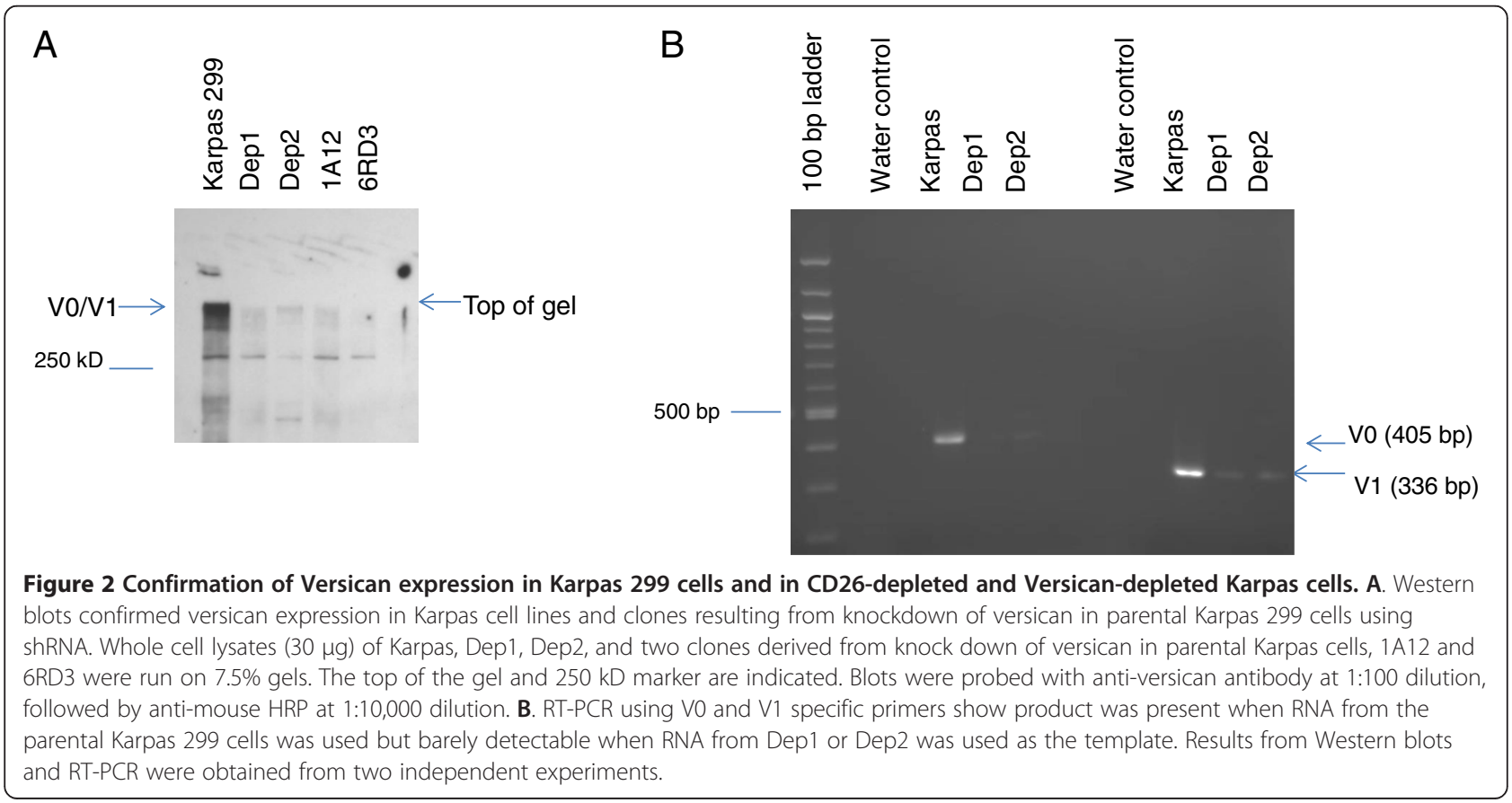




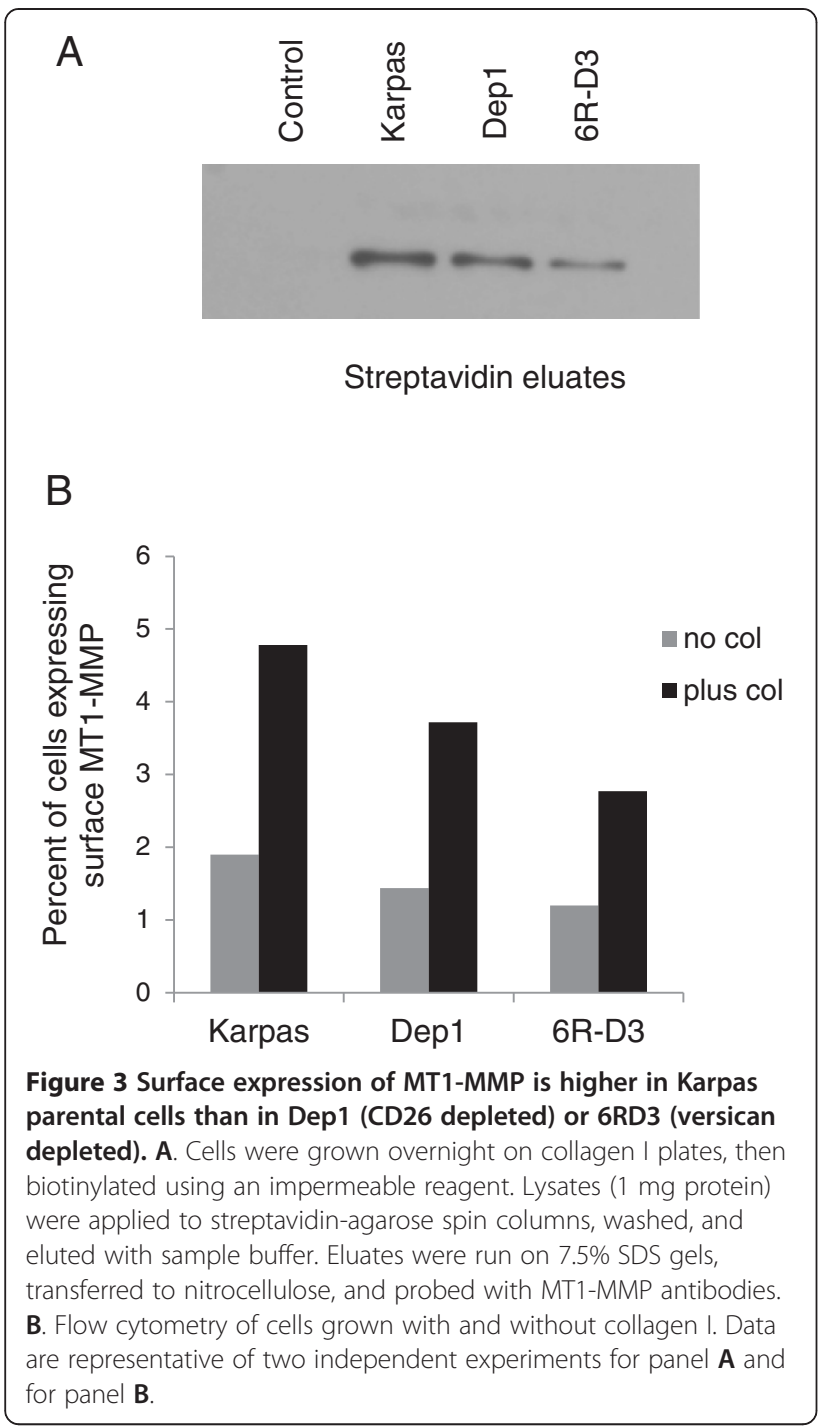

MMP to the plasma membrane [38-40], we conducted our studies in the presence or absence of PMA. In our experimental system, PMA had only a slight enhancing effect on the expression and secretion of CD44.

\section{Enhanced collagenase I activity is associated with CD26 and versican in Karpas 299 cells}

Previous work has demonstrated an association between MT1-MMP and enhanced collagen I degradation [32,41]. We next conducted two separate assays for collagenase I activity as described in Materials and Methods, one using a solid phase assay in which collagen I degradation was monitored in live cells (Figure 5A), and the other using a liquid-phase assay with vesicles isolated from conditioned media (Figure 5B). In both types of assays, parental Karpas 299 cells exhibited a higher level of collagenase I activity than Dep1 or 6RD3 clones.

\section{Adhesion to collagen I is highest in the parental Karpas 299 cell line}

Adhesion to collagen I was compared for the parental Karpas 299 cells, the CD26-depleted cells (Dep1) and versican-depleted cells (6RD3) in precoated 12 well plates. Our findings indicated that the versican-expressing parental Karpas 299 cells exhibited much greater adhesion to collagen than the versican-depleted Dep1 and 6RD3 cell lines (Figure 6).

\section{Erk(1/2) activation is highest in the parental Karpas 299 cell line}

Erk $(1 / 2)$ activation is required for CD44 [42,43] expression and cell migration and is induced by overexpression of MT1-MMP [44]. In addition, MT1-MMP expression activates Erk (1/2), which then leads to upregulation of MT1-MMP, creating a positive feedback loop [33]. To further explore the mechanism involved in MT1-MMP upregulation associated with CD26 and versican, cells
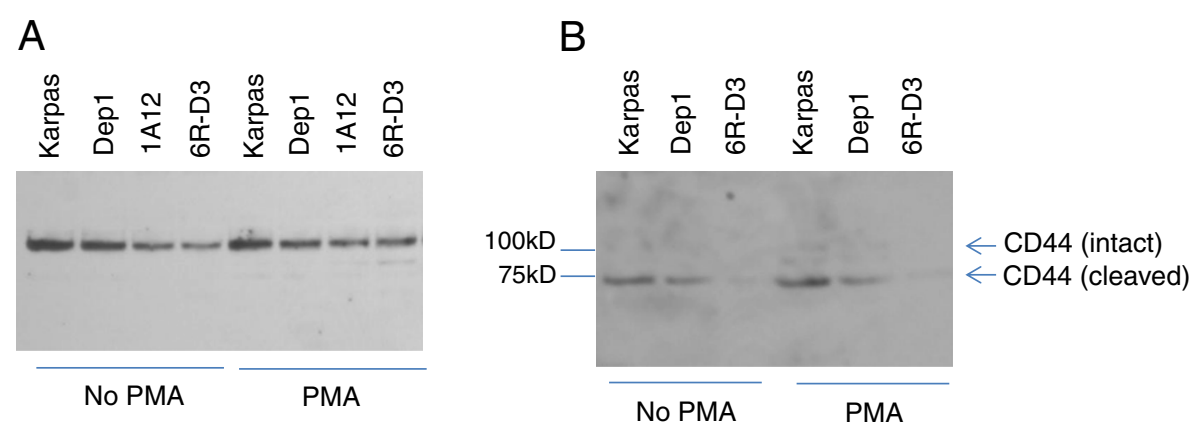

Figure 4 CD44 expression/secretion of cleaved form is higher in parental Karpas 299 cells than in Dep1 or 6RD3 cells. A. Whole cell lysates $(30 \mathrm{\mu g})$ from cells grown on collagen I plates in the presence or absence of $10 \mathrm{ng} / \mathrm{ml}$ PMA for $24 \mathrm{hr}$. B. Concentrated conditioned media $(75 \mu \mathrm{g})$ isolated from cells grown on collagen I plates for $24 \mathrm{hr}$. Samples were run on 7.5\% SDS gels, transferred, and probed with anti-CD44H, followed by anti-mouse HRP. Of note is that intact CD44 migrates as a $100 \mathrm{kD}$ protein, whereas the cleaved form migrates as a $70-75 \mathrm{kD}$ species [36,67]. Data are representative of three independent experiments. 


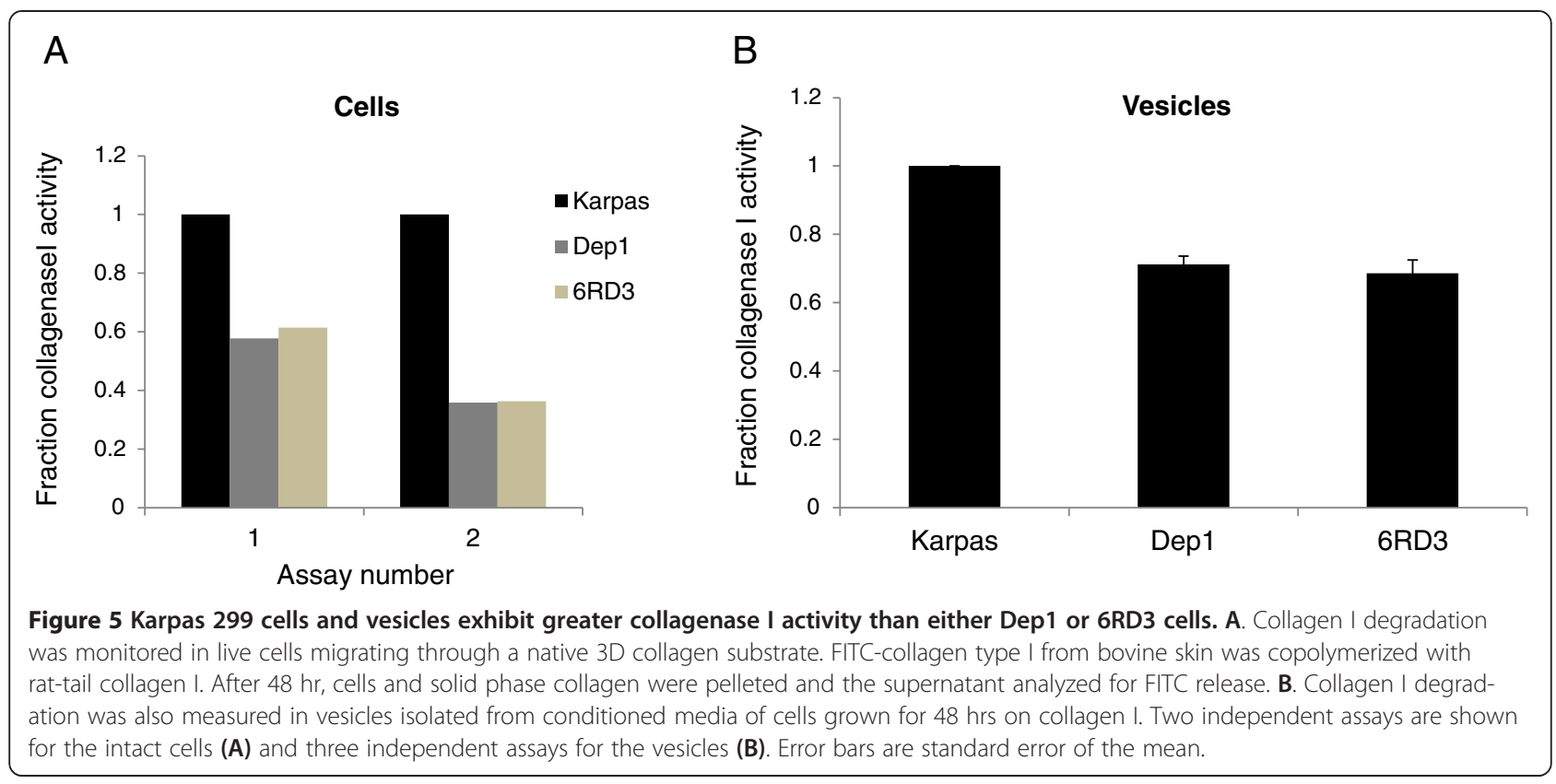

were cultured overnight in serum free medium, and the expression of MT1-MMP, phosphorylated Erk (1/2), and integrin $\alpha 5$ in vesicles isolated from the conditioned medium was determined by Western blot (Figure 7). We had previously observed that activated Erk (1/2) and MT1-MMP were present in the conditioned media (data not shown) and others have shown that MT1-MMP is present in vesicles isolated from the spent media of endothelial [45], fibrosarcoma, and melanoma cells [46]. We found that the expression of MT1-MMP was higher in parental Karpas 299 cells than in the CD26-depleted Dep1 cells or versican-depleted 6RD3 cells. Activation of
Erk (1/2) followed the same pattern, which is consistent with observations for actively migrating cells [38]. In contrast the level of the $\alpha 5$ integrin appeared to be similar in all cells.

\section{Discussion}

In this paper, we have focused on the differential expression of versican in CD26-expressing Karpas 299 cells as compared to a CD26-depleted clone and the associated changes in various cellular activities as related to tumorigenesis. As a point of reference, we presented a working model at the beginning of the paper. The emphasis is

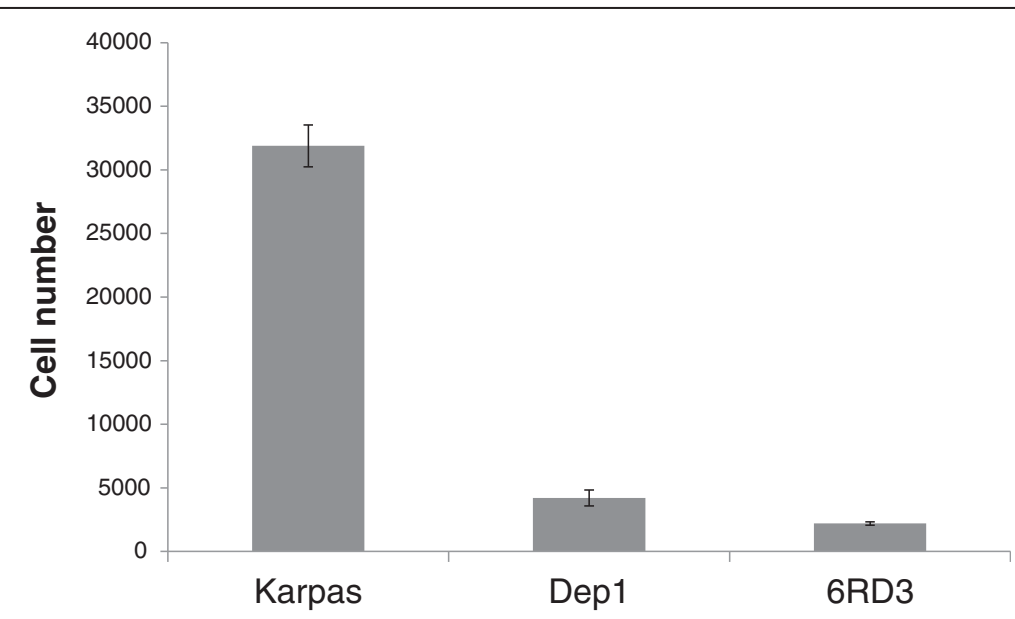

Figure 6 Adhesion assays show that Karpas 299 cells adhere to collagen I to a greater extent than CD26-depleted, Dep1, or CD26expressing, versican-depleted, 6 RD 3 cells. Cells $\left(5 \times 10^{5} /\right.$ well) were seeded into 12 well collagen I coated plates and incubated overnight. Following removal of non-adhesive cells, the cells remaining were quantified using the MTS assay. The total cell number was determined using uncoated wells and serial dilutions were used to construct a standard curve to convert absorbance at $490 \mathrm{~nm}$ to cell number. Error bars are standard error of the mean. Data are representative of three independent experiments. 


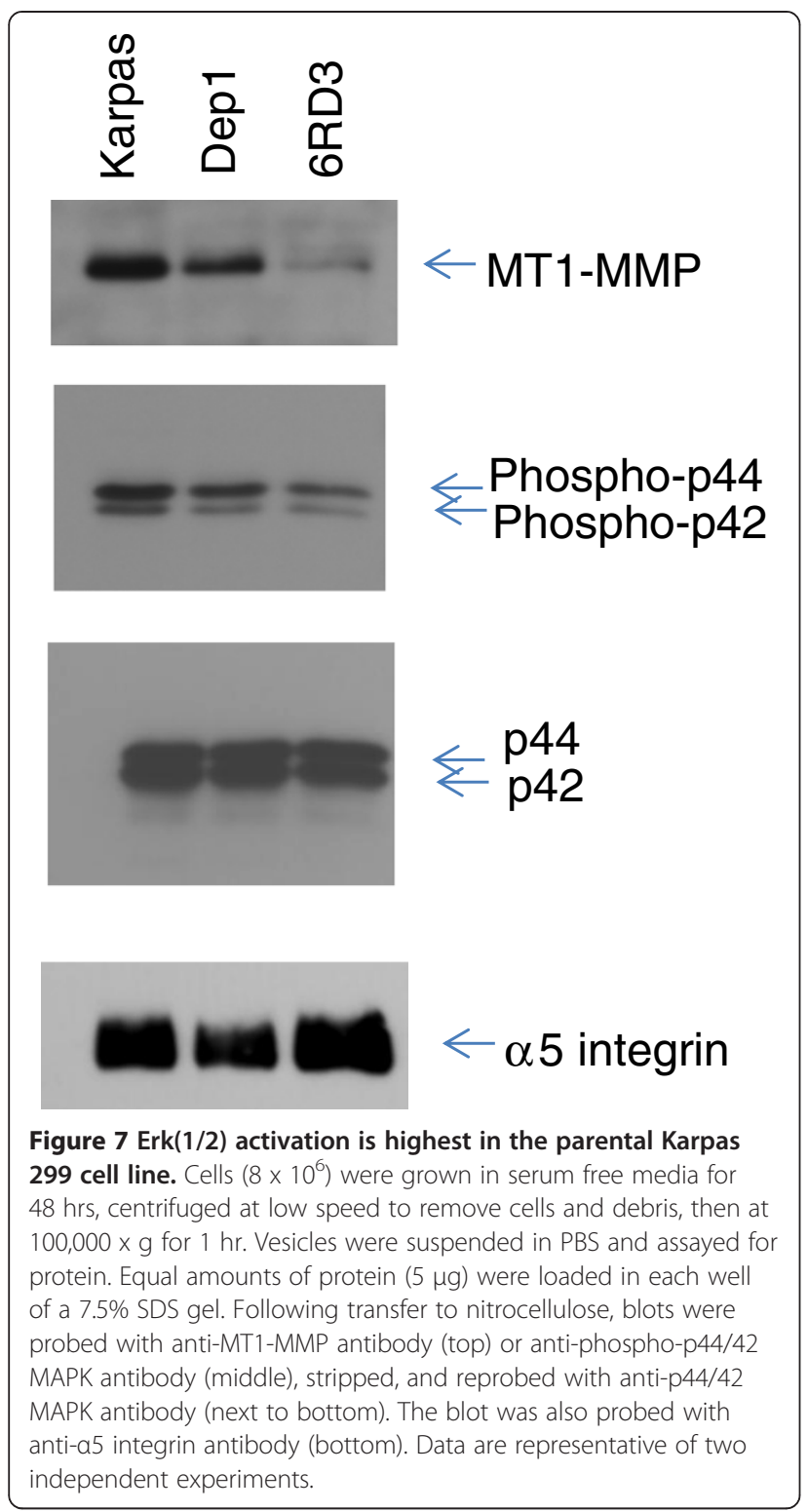

placed on MT1-MMP (MMP-14), since it is known to have several important activities which could account for the ability of CD26-expressing Karpas 299 cells to form tumors in SCID mice as opposed to the inability of CD26deficient Karpas 299 cells to develop tumors in the same animal model [8]. We do note that this simplified model does not take into account the complex roles that MT1MMP and other MMPs play in cancer progression. For example, in addition to degrading the extracellular matrix, MT1-MMP plays an important role in tumor angiogenesis [47] through upregulation of VEGF [48] and immunoregulation through its effect on the release and activation of cytokines such as TGF- $\beta$, a well-known suppressor of T-lymphocyte reaction against cancer [49].

In addition to the difference in versican expression, there were differences in adhesion, MT1-MMP surface expression, CD44 cleavage and secretion, and collagenase I activity. Although CD26 is known to bind both collagen [50,51] and fibronectin [52], versican also binds these proteins, and can further strengthen the binding of CD26-expressing cells to the extracellular matrix. This conclusion is consistent with our observation that MT1MMP surface expression was increased in cells bound to collagen I. Since localization of MT1-MMP to the cell membrane is required for its ability to degrade the extracellular matrix [32], the decreased surface expression of MT1-MMP associated with loss of versican would be predicted to have an effect on cell motility, and possibly, tumorigenesis by interfering with the ability of tumor cells to interact with the microenvironment.

Our present work also established a relationship between CD44, CD26 and versican, with CD44 cleavage/ secretion being higher in parental Karpas 299 cells than in cells depleted of versican (both CD26-depleted cells as well as CD26-expressing/versican depleted cells). Interaction with and cleavage of CD44 by MT1-MMP has been shown to facilitate migration by indirectly linking MT1MMP to the actin cytoskeleton [35,36]. The function of MT1-MMP is regulated in large part by its localization; MT1-MMP activity has been observed at invadopodia [53-55], lamellipodia [35], and focal adhesions [56], with CD44 cleavage and secretion appearing to play a role in the localization of MT1-MMP to the invadopodia [35].

Our data also indicated a higher level of ERK activation in parental Karpas 299 cells compared to CD26-depleted or CD26-expressiong/versican-depleted clones. ERK activation is required for migration, invasion $[44,57,58]$, and CD44 upregulation. The requirement for matrix proteins along with ERK activation suggests that integrins may be involved in MT1-MMP regulation [59], a conclusion that is further supported by colocalization of integrins with MT1-MMP in vesicles $[46,60]$ and the existence of common recycling pathways [61]. In a recent study, intracellular trafficking of MT1-MMP was found to be coupled with trafficking of integrin $\alpha 5$, ERK activation, and phosphorylation of MT1-MMP at $\mathrm{Thr}^{567}$ [38]. We also detected these three proteins in vesicles isolated from conditioned media; MT1-MMP and phosphorylated ERK were highest in the parental Karpas 299 cells, whereas the amount of $\alpha 5$ integrin was approximately the same in all three cell lines.

Although regulation of versican expression is not well understood, it has been shown to be a target of Wnt signaling, regulated by the phosphatidylinositol 3-kinase (PI3K) pathway in human embryonic carcinoma cells [62]. It is possible that it is also regulated by this pathway in Karpas 299 cells, since activated Akt/PKB is higher in the parental Karpas 299 cells than in CD26depleted or versican-depleted cells (unpublished observations, author). 
In addition to its ability to form homodimers, CD26 can also form heterodimers with fibroblast activation protein alpha (FAP or Seprase) [63], which shares 48\% homology with CD26 [64], but unlike CD26, can digest collagen. Although this protein complex has been detected at the invadopodia of migrating fibroblasts [65], we did not explore the role of Seprase activity in the collagenase I activity of Karpas 299 cells. However, our Western blot assays for Seprase did not detect a difference among parental Karpas 299 cells, Dep1, and 6RD3 (data not shown). While it has been suggested that CD26 and related proteins, such as FAP, may serve as valuable biomarkers for selected malignancies, better indepth understanding of the functional roles of these molecules in particular tumor types and their associated microenvironment will improve our knowledge of the implications of their expression in tumor behavior [66].

\section{Conclusions}

In summary, our data suggest that CD26 has a key role in cellular adhesion and invasion through versican and MT1-MMP expression as well as downstream signaling molecules involved in these processes. The expression of versican in Karpas 299 parental cells is likely responsible for their increased adhesion to the extracellular matrix, which is necessary for cellular interaction with ECM components and is also required for migration. The difference in the adhesiveness of the parental Karpas 299 cells and their CD26-deficient (and therefore versican deficient) counterpart, Dep1, may account for the difference in tumorigenicity previously observed in SCID mice [8].

\section{Competing interests}

The authors declare that they have no competing interests.

\section{Authors' contributions}

PAH performed the research; PAH and NHD designed the research study, analyzed the data, and wrote the paper; KO, SI and CM contributed essential reagents and analyzed the data; LHD analyzed the data and critically revised the paper. All authors read and approved the final manuscript.

\section{Acknowledgements}

We thank Neal Benson, Director of the Flow Cytometry core at the Interdisciplinary Center for Biotechnology Research at the University of Florida.

\section{Author details \\ ${ }^{1}$ Division of Hematology/Oncology, University of Florida Shands Cancer Center, Gainesville, FL 32610, USA. ²Department of Therapy Development and Innovation for Immune Disorders and Cancers, Graduate School of Medicine, Juntendo University, Tokyo 113-8421, Japan. ${ }^{3}$ Division of Hematology/Oncology, University of Florida, 1600 SW Archer Road, Box 100278, Gainesville, Florida 32610, USA.}

Received: 12 June 2013 Accepted: 30 October 2013

Published: 1 November 2013

\footnotetext{
References

1. Pang R, Law WL, Chu AC, Poon JT, Lam CS, Chow AK, Ng L, Cheung LW, Lan XR, Lan HY, et al: A subpopulation of CD26+ cancer stem cells with
}

metastatic capacity in human colorectal cancer. Cell Stem Cell 2010, 6(6):603-615.

2. de Andrade CF, Bigni R, Pombo-de-Oliveira MS, Alves G, Pereira DA: CD26/ DPPIV cell membrane expression and DPPIV activity in plasma of patients with acute leukemia. J Enzyme Inhib Med Chem 2009, 24(3):708-714.

3. De Chiara L, Rodriguez-Pineiro AM, Rodriguez-Berrocal FJ, Cordero OJ, Martinez-Ares D, Paez de la Cadena M: Serum CD26 is related to histopathological polyp traits and behaves as a marker for colorectal cancer and advanced adenomas. BMC Cancer 2010, 10:333.

4. Dohi $\mathrm{O}$, Ohtani H, Hatori M, Sato E, Hosaka M, Nagura H, Itoi E, Kokubun S: Histogenesis-specific expression of fibroblast activation protein and dipeptidylpeptidase-IV in human bone and soft tissue tumours. Histopathology 2009, 55(4):432-440

5. Varona A, Blanco L, Perez I, Gil J, Irazusta J, Lopez JI, Candenas ML, Pinto FM Larrinaga G: Expression and activity profiles of DPP IV/CD26 and NEP/ CD10 glycoproteins in the human renal cancer are tumor-type dependent. BMC Cancer 2010, 10:193.

6. Wesley UV, McGroarty M, Homoyouni A: Dipeptidyl peptidase inhibits malignant phenotype of prostate cancer cells by blocking basic fibroblast growth factor signaling pathway. Cancer Res 2005, 65(4):1325-1334.

7. Kajiyama H, Kikkawa F, Suzuki T, Shibata K, Ino K, Mizutani S: Prolonged survival and decreased invasive activity attributable to dipeptidyl peptidase IV overexpression in ovarian carcinoma. Cancer Res 2002, 62(10):2753-2757.

8. Sato T, Yamochi T, Yamochi T, Aytac U, Ohnuma K, McKee KS, Morimoto C Dang NH: CD26 regulates p38 mitogen-activated protein kinasedependent phosphorylation of integrin beta1, adhesion to extracellular matrix, and tumorigenicity of T-anaplastic large cell lymphoma Karpas 299. Cancer Res 2005, 65(15):6950-6956.

9. Ohnuma K, Yamochi T, Uchiyama M, Nishibashi K, Yoshikawa N, Shimizu N, Iwata S, Tanaka H, Dang NH, Morimoto C: CD26 up-regulates expression of CD86 on antigen-presenting cells by means of caveolin-1. Proc Natl Acad Sci USA 2004, 101(39):14186-14191.

10. Fox DA, Hussey RE, Fitzgerald KA, Acuto O, Poole C, Palley L, Daley JF, Schlossman SF, Reinherz EL: Ta1, a novel 105 KD human T cell activation antigen defined by a monoclonal antibody. J Immunol 1984, 133(3):1250-1256.

11. Dang NH, Torimoto $Y$, Deusch K, Schlossman SF, Morimoto C: Comitogenic effect of solid-phase immobilized anti-1 F7 on human CD4 T cell activation via CD3 and CD2 pathways. J Immunol 1990, 144(11):4092-4100.

12. Dang NH, Torimoto Y, Sugita K, Daley JF, Schow P, Prado C, Schlossman SF, Morimoto C: Cell surface modulation of CD26 by anti-1 F7 monoclonal antibody. Analysis of surface expression and human T cell activation. $\mathrm{J}$ Immunol 1990, 145(12):3963-3971.

13. Torimoto Y, Dang NH, Vivier E, Tanaka T, Schlossman SF, Morimoto C: Coassociation of CD26 (dipeptidyl peptidase IV) with CD45 on the surface of human T lymphocytes. J Immunol 1991, 147(8):2514-2517.

14. Carbone A, Gloghini A, Zagonel V, Aldinucci D, Gattei V, Degan M, Improta S, Sorio R, Monfardini S, Pinto A: The expression of CD26 and CD40 ligand is mutually exclusive in human T-cell non-Hodgkin's lymphomas/leukemias. Blood 1995, 86(12):4617-4626.

15. Dang NH, Aytac U, Sato K, O'Brien S, Melenhorst J, Morimoto C, Barrett AJ, Molldrem JJ: T-large granular lymphocyte lymphoproliferative disorder: expression of CD26 as a marker of clinically aggressive disease and characterization of marrow inhibition. Br J Haematol 2003, 121(6):857-865.

16. Yamaguchi U, Nakayama R, Honda K, Ichikawa H, Hasegawa T, Shitashige M, Ono M, Shoji A, Sakuma T, Kuwabara H, et al: Distinct gene expressiondefined classes of gastrointestinal stromal tumor. J Clin Oncol 2008, 26(25):4100-4108.

17. Inamoto T, Yamada T, Ohnuma K, Kina S, Takahashi N, Yamochi T, Inamoto S, Katsuoka Y, Hosono O, Tanaka H, et al: Humanized anti-CD26 monoclonal antibody as a treatment for malignant mesothelioma tumors. Clin Cancer Res 2007, 13(14):4191-4200.

18. Droz D, Zachar D, Charbit L, Gogusev J, Chretein Y, Iris L: Expression of the human nephron differentiation molecules in renal cell carcinomas. Am J Pathol 1990, 137(4):895-905.

19. Le Naour F, Andre M, Greco C, Billard M, Sordat B, Emile J-F, Lanza F, Boucheix C, Rubinstein E: Profiling of the Tetraspanin web of human colon cancer cells. Mol Cell Proteomics 2006, 5(5):845-857.

20. Stange T, Kettmann U, Holzhausen HJ: Immunoelectron microscopic demonstration of the membrane proteases aminopeptidase N/CD13 and 
dipeptidyl peptidase IV/CD26 in normal and neoplastic renal parenchymal tissues and cells. Eur J Histochem 2000, 44(2):157-164.

21. Javidroozi M, Zucker S, Chen WT: Plasma seprase and DPP4 levels as markers of disease and prognosis in cancer. Dis Markers 2012, 32(5):309-320

22. Havre PA, Abe M, Urasaki Y, Ohnuma K, Morimoto C, Dang NH: CD26 expression on T cell lines increases SDF-1-alpha-mediated invasion. Br J Cancer 2009, 101(6):983-991.

23. Wight TN: Versican: a versatile extracellular matrix proteoglycan in cell biology. Curr Opin Cell Biol 2002, 14(5):617-623.

24. Yamagata M, Yamada KM, Yoneda M, Suzuki S, Kimata K: Chondroitin sulfate proteoglycan (PG-M-like proteoglycan) is involved in the binding of hyaluronic acid to cellular fibronectin. J Biol Chem 1986, 261(29):13526-13535.

25. Theocharis AD: Versican in health and disease. Connect Tissue Res 2008, 49(3):230-234

26. Wu YJ, La Pierre DP, Wu J, Yee AJ, Yang BB: The interaction of versican with its binding partners. Cell Res 2005, 15(7):483-494.

27. Ricciardelli C, Sakko AJ, Ween MP, Russell DL, Horsfall DJ: The biological role and regulation of versican levels in cancer. Cancer Metastasis Rev 2009, 28(1-2):233-245.

28. Theocharis AD, Skandalis SS, Tzanakakis GN, Karamanos NK: Proteoglycans in health and disease: novel roles for proteoglycans in malignancy and their pharmacological targeting. FEBS J 2010, 277(19):3904-3923.

29. Cattaruzza S, Schiappacassi M, Ljungberg-Rose A, Spessotto P, Perissinotto D, Morgelin M, Mucignat MT, Colombatti A, Perris R: Distribution of PG-M/ versican variants in human tissues and de novo expression of isoform V3 upon endothelial cell activation, migration, and neoangiogenesis in vitro. J Biol Chem 2002, 277(49):47626-47635.

30. Dolo V, Adobati E, Canevari S, Picone MA, Vittorelli ML: Membrane vesicles shed into the extracellular medium by human breast carcinoma cells carry tumor-associated surface antigens. Clin Exp Metastasis 1995, 13(4):277-286.

31. Wolf K, Muller R, Borgmann S, Brocker EB, Friedl P: Amoeboid shape change and contact guidance: T-lymphocyte crawling through fibrillar collagen is independent of matrix remodeling by MMPs and other proteases. Blood 2003, 102(9):3262-3269.

32. Sabeh F, Ota I, Holmbeck K, Birkedal-Hansen H, Soloway P, Ballbin M, Lopez-Otin C, Shapiro S, Inada M, Krane S, et al: Tumor cell traffic through the extracellular matrix is controlled by the membrane-anchored collagenase MT1-MMP. J Cell Biol 2004, 167(4):769-781.

33. Takino T, Miyamori H, Watanabe $Y$, Yoshioka K, Seiki M, Sato H: Membrane type 1 matrix metalloproteinase regulates collagen-dependent mitogenactivated protein/extracellular signal-related kinase activation and cell migration. Cancer Res 2004, 64(3):1044-1049.

34. Gingras D, Beliveau R: Emerging concepts in the regulation of membranetype 1 matrix metalloproteinase activity. Biochim Biophys Acta 2010, 1803(1):142-150.

35. Mori H, Tomari T, Koshikawa N, Kajita M, Itoh Y, Sato H, Tojo H, Yana I, Seiki M: CD44 directs membrane-type 1 matrix metalloproteinase to lamellipodia by associating with its hemopexin-like domain. EMBO J 2002, 21(15):3949-3959.

36. Kajita M, Itoh Y, Chiba T, Mori H, Okada A, Kinoh H, Seiki M: Membranetype 1 matrix metalloproteinase cleaves CD44 and promotes cell migration. J Cell Biol 2001, 153(5):893-904.

37. Ladeda V, Aguirre Ghiso JA, Bal de Kier Joffe E: Function and expression of CD44 during spreading, migration, and invasion of murine carcinoma cells. Exp Cell Res 1998, 242(2):515-527.

38. Williams KC, Coppolino MG: Phosphorylation of membrane type 1-matrix metalloproteinase (MT1-MMP) and its vesicle-associated membrane protein 7 (VAMP7)-dependent trafficking facilitate cell invasion and migration. J Biol Chem 2011, 286(50):43405-43416.

39. Zucker S, Hymowitz M, Conner CE, DiYanni EA, Cao J: Rapid trafficking of membrane type 1-matrix metalloproteinase to the cell surface regulates progelatinase a activation. Lab Invest 2002, 82(12):1673-1684

40. Kean MJ, Williams KC, Skalski M, Myers D, Burtnik A, Foster D, Coppolino MG: VAMP3, syntaxin-13 and SNAP23 are involved in secretion of matrix metalloproteinases, degradation of the extracellular matrix and cell invasion. J Cell Sci 2009, 122(Pt 22):4089-4098

41. Evans RD, Itoh Y: Analyses of MT1-MMP activity in cells. Methods Mol Med 2007, 135:239-249.
42. Judd NP, Winkler AE, Murillo-Sauca O, Brotman JJ, Law JH, Lewis JS Jr, Dunn GP, Bui JD, Sunwoo JB, Uppaluri R: ERK1/2 regulation of CD44 modulates oral cancer aggressiveness. Cancer Res 2012, 72(1):365-374.

43. Tanimura S, Asato K, Fujishiro SH, Kohno M: Specific blockade of the ERK pathway inhibits the invasiveness of tumor cells: down-regulation of matrix metalloproteinase-3/-9/-14 and CD44. Biochem Biophys Res Commun 2003, 304(4):801-806.

44. Gingras D, Bousquet-Gagnon N, Langlois S, Lachambre MP, Annabi B, Beliveau R: Activation of the extracellular signal-regulated protein kinase (ERK) cascade by membrane-type-1 matrix metalloproteinase (MT1-MMP). FEBS Lett 2001, 507(2):231-236.

45. Taraboletti G, D'Ascenzo S, Borsotti P, Giavazzi R, Pavan A, Dolo V: Shedding of the matrix metalloproteinases MMP-2, MMP-9, and MT1-MMP as membrane vesicle-associated components by endothelial cells. Am J Pathol 2002, 160(2):673-680

46. Hakulinen J, Sankkila L, Sugiyama N, Lehti K, Keski-Oja J: Secretion of active membrane type 1 matrix metalloproteinase (MMP-14) into extracellular space in microvesicular exosomes. J Cell Biochem 2008, 105(5):1211-1218.

47. Chun TH, Sabeh F, Ota I, Murphy H, McDonagh KT, Holmbeck K, BirkedalHansen $H$, Allen ED, Weiss SJ: MT1-MMP-dependent neovessel formation within the confines of the three-dimensional extracellular matrix. J Cell Biol 2004, 167(4):757-767.

48. Sounni NE, Devy L, Hajitou A, Frankenne F, Munaut C, Gilles C, Deroanne C Thompson EW, Foidart JM, Noel A: MT1-MMP expression promotes tumor growth and angiogenesis through an up-regulation of vascular endothelial growth factor expression. FASEB J 2002, 16(6):555-564.

49. Gialeli $C$, Theocharis AD, Karamanos NK: Roles of matrix metalloproteinases in cancer progression and their pharmacological targeting. FEBS J 2011, 278(1):16-27.

50. Dang NH, Torimoto $Y$, Schlossman SF, Morimoto C: Human CD4 helper T cell activation: functional involvement of two distinct collagen receptors, 1 F7 and VLA integrin family. J Exp Med 1990, 172(2):649-652.

51. Loster K, Zeilinger K, Schuppan D, Reutter W: The cysteine-rich region of dipeptidyl peptidase IV (CD 26) is the collagen-binding site. Biochem Biophys Res Commun 1995, 217(1):341-348.

52. Cheng $\mathrm{HC}$, Abdel-Ghany M, Pauli BU: A novel consensus motif in fibronectin mediates dipeptidyl peptidase IV adhesion and metastasis. J Biol Chem 2003, 278(27):24600-24607.

53. Artym W, Zhang Y, Seillier-Moiseiwitsch F, Yamada KM, Mueller SC: Dynamic interactions of cortactin and membrane type 1 matrix metalloproteinase at invadopodia: defining the stages of invadopodia formation and function. Cancer Res 2006, 66(6):3034-3043.

54. Clark ES, Weaver AM: A new role for cortactin in invadopodia: regulation of protease secretion. Eur J Cell Biol 2008, 87(8-9):581-590.

55. Nakahara H, Howard L, Thompson EW, Sato H, Seiki M, Yeh Y, Chen WT: Transmembrane/cytoplasmic domain-mediated membrane type 1-matrix metalloprotease docking to invadopodia is required for cell invasion. Proc Natl Acad Sci USA 1997, 94(15):7959-7964.

56. Wang $Y$, McNiven MA: Invasive matrix degradation at focal adhesions occurs via protease recruitment by a FAK-p130Cas complex. J Cell Biol 2012, 196(3):375-385.

57. Tague SE, Muralidharan V, D'Souza-Schorey C: ADP-ribosylation factor 6 regulates tumor cell invasion through the activation of the MEK/ERK signaling pathway. Proc Natl Acad Sci USA 2004, 101(26):9671-9676.

58. Takino T, Tsuge H, Ozawa T, Sato H: MT1-MMP promotes cell growth and ERK activation through c-Src and paxillin in three-dimensional collagen matrix. Biochem Biophys Res Commun 2010, 396(4):1042-1047.

59. Howe AK, Aplin AE, Juliano RL: Anchorage-dependent ERK signalingmechanisms and consequences. Curr Opin Genet Dev 2002, 12(1):30-35.

60. Gonzalo P, Moreno V, Galvez BG, Arroyo AG: MT1-MMP and integrins: hand-to-hand in cell communication. Biofactors 2010, 36(4):248-254.

61. Ramsay AG, Marshall JF, Hart IR: Integrin trafficking and its role in cancer metastasis. Cancer Metastasis Rev 2007, 26(3-4):567-578.

62. Rahmani M, Read JT, Carthy JM, McDonald PC, Wong BW, Esfandiarei M, Si $X$, Luo Z, Luo H, Rennie PS, et al: Regulation of the versican promoter by the beta-catenin-T-cell factor complex in vascular smooth muscle cells. J Biol Chem 2005, 280(13):13019-13028.

63. Ghersi G, Zhao Q, Salamone M, Yeh Y, Zucker S, Chen WT: The protease complex consisting of dipeptidyl peptidase IV and Seprase plays a role in the migration and invasion of human endothelial cells in collagenous matrices. Cancer Res 2006, 66(9):4652-4661. 
64. Scanlan MJ, Raj BK, Calvo B, Garin-Chesa P, Sanz-Moncasi MP, Healey JH, Old $\sqcup$, Rettig WJ: Molecular cloning of fibroblast activation protein alpha, a member of the serine protease family selectively expressed in stromal fibroblasts of epithelial cancers. Proc Natl Acad Sci USA 1994, 91(12):5657-5661.

65. Ghersi G, Dong H, Goldstein LA, Yeh Y, Hakkinen L, Larjava HS, Chen WT: Regulation of fibroblast migration on collagenous matrix by a cell surface peptidase complex. J Biol Chem 2002, 277(32):29231-29241.

66. Sedo A, Stremenova J, Busek P, Duke-Cohan JS: Dipeptidyl peptidase-IV and related molecules: markers of malignancy? Exp Opin Med Diagn 2008, 2(6):677-689.

67. Suenaga $\mathrm{N}$, Mori $\mathrm{H}$, Itoh $\mathrm{Y}$, Seiki M: CD44 binding through the hemopexinlike domain is critical for its shedding by membrane-type 1 matrix metalloproteinase. Oncogene 2005, 24(5):859-868.

doi:10.1186/1471-2407-13-517

Cite this article as: Havre et al:: CD26 Expression on T-Anaplastic Large Cell Lymphoma (ALCL) Line Karpas 299 is associated with increased expression of Versican and MT1-MMP and enhanced adhesion. BMC Cancer 2013 13:517.

\section{Submit your next manuscript to BioMed Central and take full advantage of:}

- Convenient online submission

- Thorough peer review

- No space constraints or color figure charges

- Immediate publication on acceptance

- Inclusion in PubMed, CAS, Scopus and Google Scholar

- Research which is freely available for redistribution 